\title{
Role of the Intramolecular Phase in High-Harmonic Generation
}

\author{
M. Lein, N. Hay, R. Velotta,* J.P. Marangos, and P. L. Knight \\ Blackett Laboratory, Imperial College of Science, Technology and Medicine, London SW7 2BW, United Kingdom
}

(Received 6 November 2001; published 23 April 2002)

\begin{abstract}
We study numerically the generation of high-order harmonics by two-center molecules for arbitrary angles between the molecular axis and the laser polarization axis. For fixed angle, the harmonic spectrum exhibits a minimum at a frequency which is independent of the laser parameters. The amplitude of each harmonic is strongly angle dependent, and a pronounced minimum is found at the same angle where a sudden jump in the harmonic phase occurs. By calculating the spatial dependence of the harmonic amplitudes and phases, we are able to explain these effects in terms of interfering contributions from various regions within the molecule.
\end{abstract}

DOI: $10.1103 /$ PhysRevLett.88.183903

The interaction of strong laser pulses with atoms or molecules leads to the generation of high-order harmonics [1-4]. This process is one of the most studied aspects of strong-field physics because it serves as a source of coherent radiation at high frequencies. Nevertheless, most of the experimental and theoretical work has been devoted to atoms, and, in contrast, high-harmonic generation by molecules still leaves open many questions.

The production of high harmonics in atoms is well explained by a recollision mechanism [5]: The strong electric field ionizes the atom or molecule and thus creates a free electron. The electron is then accelerated by the oscillating field and may be driven back to the core if the laser pulse is linearly polarized. A high-energy photon is emitted if the recollision with the core leads to recombination. Other than radiation, recollisions can also result in elastic backscattering [6] or double ionization [5]. In the case of atoms, a maximum kinetic energy of $3.17 U_{\mathrm{p}}$ for the returning electron is predicted, leading to a cutoff at a photon energy of $I_{\mathrm{p}}+3.17 U_{\mathrm{p}}$. Here, $U_{\mathrm{p}}=E_{0}^{2} /\left(4 \omega^{2}\right)$ is the ponderomotive potential in the laser field of amplitude $E_{0}$ and frequency $\omega$, and $I_{\mathrm{p}}$ is the ionization potential. By its simplicity, the recollision picture is extremely useful for purposes of interpretation and will be utilized to explain our results below.

Previous theoretical work on two-center molecules has pointed towards interesting effects which are specific to molecules: Early work has demonstrated the importance of charge-resonance effects [7]. Further, an electron can be emitted at one center and recombine at the other center, giving rise to new cutoff laws $[8,9]$. Also, it was demonstrated that non-Born-Oppenheimer dynamics becomes relevant in the case of oriented isotopically asymmetric molecules [10]. Particularly related to the present work are earlier model calculations which showed that the harmonic yield depends on the orientation of the molecule with respect to the electric field $[8,11]$. Reference [8] concentrated mainly on internuclear separations above the equilibrium distance. For molecules at their equilibrium distance, Refs. $[8,11]$ compared only between parallel and perpendicular alignment of the molecules relative to
PACS numbers: 42.65.Ky, 33.80.Rv

the field. Furthermore, the orientation dependence of the harmonic phase has not been investigated.

Experiments have shown that harmonic generation is enhanced when the molecules are prealigned (using a pulse below the intensity threshold for ionization and high-harmonic generation) [12], at least if the harmonicgeneration process itself is not complicated by reorientation during the action of the intense pulse. In these experiments, the molecules were aligned either parallel or perpendicular to the linearly polarized intense pulse.

It is likely that experimenters will make further efforts to increase the high-harmonic output by varying the angle of alignment continuously between $0^{\circ}$ and $90^{\circ}$. Therefore, in this paper we explore the dependence of harmonic generation on this angle (using linearly polarized laser pulses throughout). For various fixed orientations of the molecular axis, we calculate the harmonic amplitudes as well as the harmonic phases. One of our main results is the appearance of a pronounced interference minimum, observable either in the harmonic spectrum at a fixed angle or in the yield of a particular harmonic as a function of the angle. In the latter case, the harmonic phase changes dramatically in the angular range around the interference minimum. This leads to phase mismatching and thereby to a severe suppression of the harmonic yield when contributions of different angles are superimposed, as it happens in an ensemble of randomly oriented molecules. Taking these effects into account, it will be possible to maximize or minimize specific harmonics by controlled alignment.

We employ two-dimensional (2D) models of the $\mathrm{H}_{2}{ }^{+}$ molecular ion and the $\mathrm{H}_{2}$ molecule. A direct comparison to experiment is hampered by the fact that such light molecules are reoriented by a short intense pulse precluding controllable alignment. However, the numerically observed interference effect appears to be a universal phenomenon which will also occur in heavier molecules. These are hardly reoriented by an intense femtosecond pulse, facilitating controllable alignment.

In the $2 \mathrm{D} \mathrm{H}_{2}^{+}$model, the two nuclei are fixed at an internuclear distance of 2 a.u. For the interaction between the electron and the nuclei, we employ the soft-Coulomb 
potential,

$$
V(x, y)=-\sum_{k=1,2} 1 / \sqrt{\left(x-x_{k}\right)^{2}+\left(y-y_{k}\right)^{2}+\epsilon},
$$

where $\left(x_{1}, y_{1}\right)$ and $\left(x_{2}, y_{2}\right)$ are the positions of the nuclei. The smoothing parameter $\epsilon$ is set to 0.5 in order to reproduce the electronic ground-state energy of $-30 \mathrm{eV}$. (This value does not include the Coulomb repulsion between the nuclei.)

In the 2D model of $\mathrm{H}_{2}$, we have two nuclei at a distance of 1.4 a.u. This system is treated within the Hartee-Fock approximation. Using a soft-Coulomb electron-electron interaction, the effective potential (without laser field) becomes

$$
\begin{aligned}
V_{\mathrm{eff}}(x, y, t)= & V(x, y) \\
& +\int \frac{|\varphi(\tilde{x}, \tilde{y}, t)|^{2} d \tilde{x} d \tilde{y}}{\sqrt{(x-\tilde{x})^{2}+(y-\tilde{y})^{2}+\eta}},
\end{aligned}
$$

where $\varphi(x, y, t)$ is the time-dependent single-electron wave function. With $\epsilon=0.41$ and $\eta=0.36$, both the electronic ground-state energy $(-51 \mathrm{eV})$ and the "vertical" ionization potential $(16 \mathrm{eV})$ are in agreement with the real values for $\mathrm{H}_{2}$ [13].

After the ground state is found by propagation in imaginary time, the wave function is propagated in real time under the influence of the laser pulse. The electric field is switched on and off linearly over three optical cycles, and the total pulse duration is ten cycles. Employing a wavelength of $780 \mathrm{~nm}$, this corresponds to a pulse duration of $26 \mathrm{fs}$. The interaction between molecule and laser is treated within the dipole approximation using the velocity gauge. The direction of the electric field is taken along the $x$ axis: $\mathbf{E}(t)=(E(t), 0,0)$. The time-dependent Schrödinger equation,

$$
i \frac{\partial}{\partial t} \varphi(\mathbf{r}, t)=\left(\frac{\mathbf{p}^{2}}{2}+p_{x} A(t)+V(\mathbf{r})\right) \varphi(\mathbf{r}, t),
$$

is solved on a grid of at least $280 \times 70$ a.u. size by means of the split-operator method [14] with 2048 time steps per optical cycle. Here, $A(t)=-\int_{0}^{t} E\left(t^{\prime}\right) d t^{\prime}$, and the usual term proportional to $A(t)^{2}$ has been eliminated by a unitary transformation as we assume a spatially independent field. For $\mathrm{H}_{2}$, the potential $V$ has to be replaced by $V_{\text {eff }}$. We note that the spectra presented in this work remain unchanged when a larger or finer grid is used.

The coherent part of the harmonic spectrum is obtained from the Fourier transformed dipole acceleration expectation value [15]:

$$
\begin{aligned}
S_{\hat{\mathbf{e}}}(\omega) & \sim|\hat{\mathbf{e}} \cdot \mathbf{a}(\omega)|^{2} \\
& =\left|\int\langle\varphi(t)|\hat{\mathbf{e}} \cdot(\nabla V+\mathbf{E}(t))| \varphi(t)\rangle e^{i \omega t} d t\right|^{2},
\end{aligned}
$$

where $\hat{\mathbf{e}}$ is a unit vector along the polarization axis of interest. In Eq. (4), the accelerated motion of the center of mass is neglected for $\mathrm{H}_{2}{ }^{+}$.
In an ensemble of randomly oriented molecules, destructive interference between the contributions from various orientations leads to $S_{\hat{\mathbf{e}}}=0$ if $\hat{\mathbf{e}}$ is perpendicular to the laser field. This component also vanishes in an aligned ensemble of molecules if the molecules are aligned parallel or perpendicular to the laser field as in the experiment of Ref. [12].

Guided by the notion that the harmonics polarized parallel to the incoming field are the more important ones, we show a collection of such spectra for $2 \mathrm{D} \mathrm{H}_{2}{ }^{+}$ in Figs. 1(a)-1(c), 1(e), and 1(f). The laser intensities are $5 \times 10^{14} \mathrm{~W} / \mathrm{cm}^{2}$ and $1 \times 10^{15} \mathrm{~W} / \mathrm{cm}^{2}$ as indicated. Well-defined plateaus and clear cutoffs are found in all spectra. The cutoff positions agree well with the $I_{\mathrm{p}}+$ $3.17 U_{\mathrm{p}}$ law which predicts maximum harmonic orders of 75 and 133, respectively. The most remarkable feature of these graphs is a minimum at a harmonic frequency which increases as we increase the angle between the molecule and the electric field. The location of the minimum is indicated by an arrow in each panel. (The precise position is best found by plotting the data as in Fig. 3.) For the higher intensity, the minimum is not as pronounced as for the lower intensity. Nonetheless, a comparison between the different angles clearly shows for both intensities a dip moving through the harmonic spectrum as the angle is increased. It is remarkable that the position of the minima is the same for both intensities. Moreover, calculations for the smaller laser wavelength $390 \mathrm{~nm}$ (not shown here) give minima at almost the same harmonic frequencies. Hence, we conclude that the minimum appears at a harmonic frequency which is independent of the laser parameters and thus characterizes the molecular species.

The spectra for the 2D $\mathrm{H}_{2}$ molecule in Fig. 2 exhibit the same phenomenon. However, at the same angle of
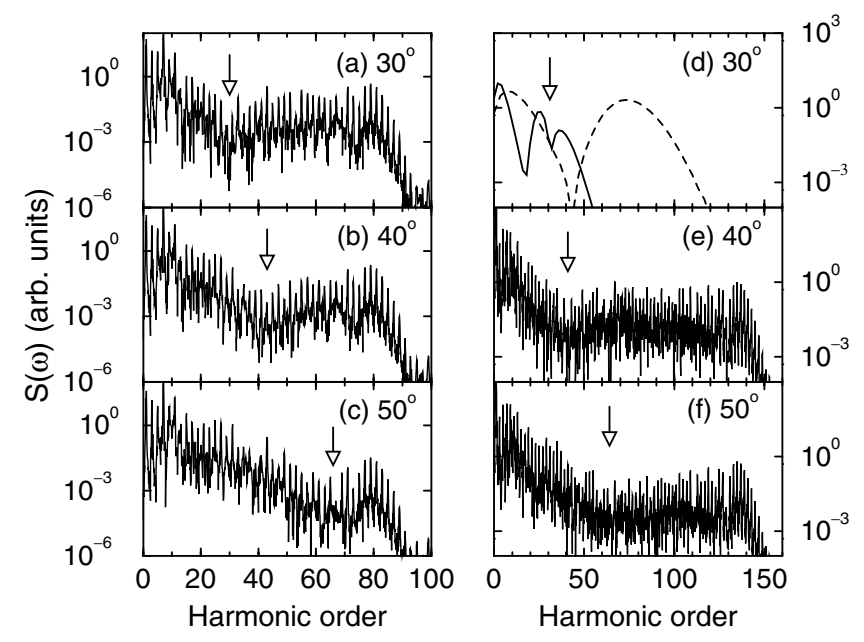

FIG. 1. Spectra of harmonics polarized parallel to the laser field for $2 \mathrm{D}_{2}{ }^{+}$. The angle between the molecular axis and the polarization axis is as indicated. Left: $780 \mathrm{~nm}$ pulses of $5 \times 10^{14} \mathrm{~W} / \mathrm{cm}^{2}$ intensity. Panel (d): Recollisions corresponding to the 31st (solid line) and 75th harmonic (dashed line). Panels (e),(f): $780 \mathrm{~nm}$ pulses of $1 \times 10^{15} \mathrm{~W} / \mathrm{cm}^{2}$ intensity. The arrows point out the position of the interference minimum. 


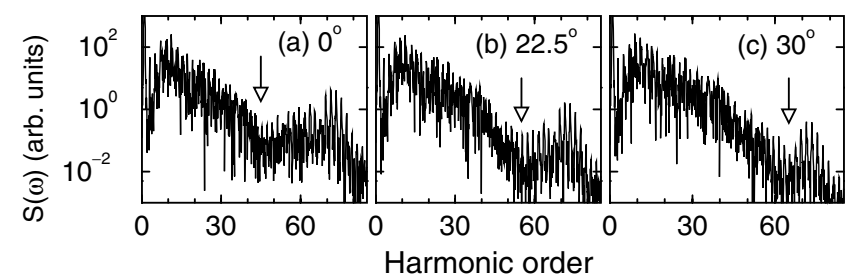

FIG. 2. Same as Fig. 1, but for the $2 \mathrm{D}_{2}$ molecule. The laser intensity is $5 \times 10^{14} \mathrm{~W} / \mathrm{cm}^{2}$.

alignment, the minimum is located at a higher harmonic frequency. This result confirms that the location of the minimum depends on the molecule. Here, we do not show results for $10^{15} \mathrm{~W} / \mathrm{cm}^{2}$ since the molecule would be completely ionized within a few optical cycles.

Figures 1 and 2 suggest that an individual peak in the harmonic spectrum should pass through a minimum as the angle of alignment is varied. Furthermore, the angle at which the minimum is located should become larger with increasing harmonic order. Indeed, this is confirmed by the numerical results shown in panels 3(a) and 3(c), where we have plotted the harmonic amplitude versus the angle of alignment. The amplitude is obtained by taking the square root of Eq. (4). For both $2 \mathrm{D} \mathrm{H}_{2}{ }^{+}$and $2 \mathrm{D} \mathrm{H}_{2}$, we find that the higher-order harmonic (dashed line) assumes its minimum at a larger angle than the lower-order harmonic (solid line).

Identifying the phase of the complex integral in Eq. (4) as the phase of the emitted harmonic radiation allows us to calculate also the dependence of the harmonic phase on the angle of alignment. The results are shown in panels 3(b) and 3(d). Interestingly, the phase changes rather slowly as the angle is varied except for a critical angle where a sudden jump in the phase occurs for parallel polarization. Apparently, this is the same angle where the amplitude passes through a minimum. Furthermore, the numerical results suggest that the phase jumps by a value close to $\pi$. (For $\mathrm{H}_{2}$, the size of the jump is somewhat smaller and the
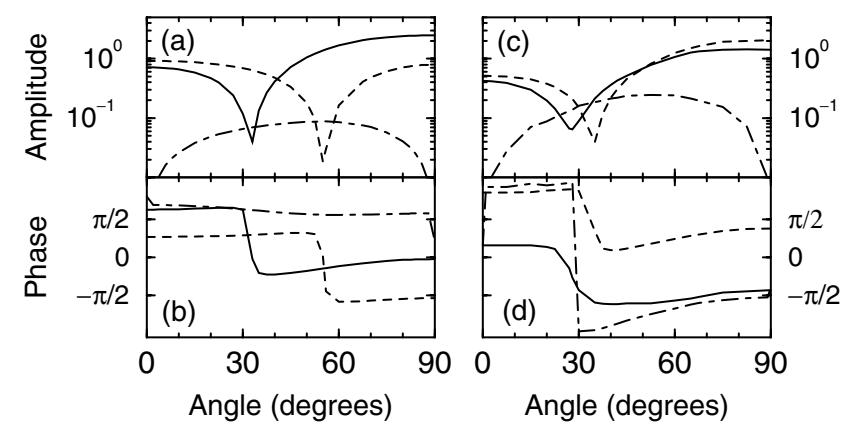

FIG. 3. Harmonic amplitude and phase versus angle of alignment for $780 \mathrm{~nm}$ pulses of $5 \times 10^{14} \mathrm{~W} / \mathrm{cm}^{2}$ intensity. Left: $2 \mathrm{D}$ $\mathrm{H}_{2}{ }^{+}$molecular ion; 31st/79th harmonic (solid line/dashed line) of parallel polarization and 79th harmonic of perpendicular polarization (dot-dashed line). Right: 2D $\mathrm{H}_{2}$ molecule; 61st/71st harmonic (solid line/dashed line) of parallel polarization and 71 st harmonic of perpendicular polarization (dot-dashed line). transition appears to be smoother in this case.) The implication for an ensemble of randomly oriented molecules is obvious: Angles below the critical angle give contributions which interfere destructively with those from angles above the critical value. The total harmonic yield will then be significantly smaller than that of an aligned ensemble of molecules, as it was experimentally observed in Ref. [12].

For completeness, we also show examples of the radiation polarized perpendicularly to the laser pulse. These are the dot-dashed curves in Fig. 3. For most angles of alignment, the amplitude is much lower than that of parallel polarization. At $0^{\circ}$ and $90^{\circ}$, it vanishes for symmetry reasons. For the intermediate angles, we find neither a pronounced minimum in the amplitude nor a sudden jump in the phase. Note that, for $\mathrm{H}_{2}$, the behavior of the phase around $30^{\circ}$ is not a phase jump but merely a consequence of the fact that the phase is defined only up to an additive constant $2 \pi n$, where $n$ is an integer.

In the following, we explain the mechanism behind these phenomena. As a first step, we show that they can be understood in terms of the recollision picture. To that end, we perform a numerical experiment, where an electron wave packet collides with a molecular core. To be precise, the initial state is chosen to be a superposition of a Gaussian wave packet $\varphi_{c}(\mathbf{r}, t)$, moving towards the two nuclei, and the ground-state wave function $\varphi_{0}(\mathbf{r})$ :

$$
\varphi(\mathbf{r}, t=0)=\left(\varphi_{0}(\mathbf{r})+\varphi_{c}(\mathbf{r}, t=0)\right) / \sqrt{2} .
$$

From the time evolution of $\varphi(\mathbf{r}, t)$, we calculate the radiation emitted during the collision. In this calculation, the laser is switched off. In Fig. 1(d), we show the result for an angle of $30^{\circ}$ between the molecular axis and the initial direction of wave-packet motion. The energy difference between the continuum wave packet and the ground state equals the photon energy of the 31st harmonic for the solid line and the 75th harmonic for the dashed line. The broad maxima at frequencies below the 20th/45th order for the solid/dashed curve stem from the accelerated motion of the continuum wave packet itself (continuum-continuum transitions). We are more interested in the radiation around the 31st/75th harmonic which corresponds to the possible transition of an electron from a continuum state to the ground state. The collision with higher impact energy gives rise to a structureless hump centered at the 75th harmonic. In contrast, the collision with the lower impact energy generates a double-peak structure around the 31st harmonic. A minimum occurs at the 31st harmonic although the impact energy is chosen such that this harmonic frequency could be produced with high probability. It is important to note that the minimum is located at almost the same frequency as in the harmonic spectrum generated under the influence of a full laser pulse, Fig. 1(a). This is a clear proof that the physical process leading to the suppression of certain harmonics is contained in the quantum mechanical recollision. The mechanism of suppression occurs independent of how the electron was promoted into the continuum prior to the recollision. For this reason, the 


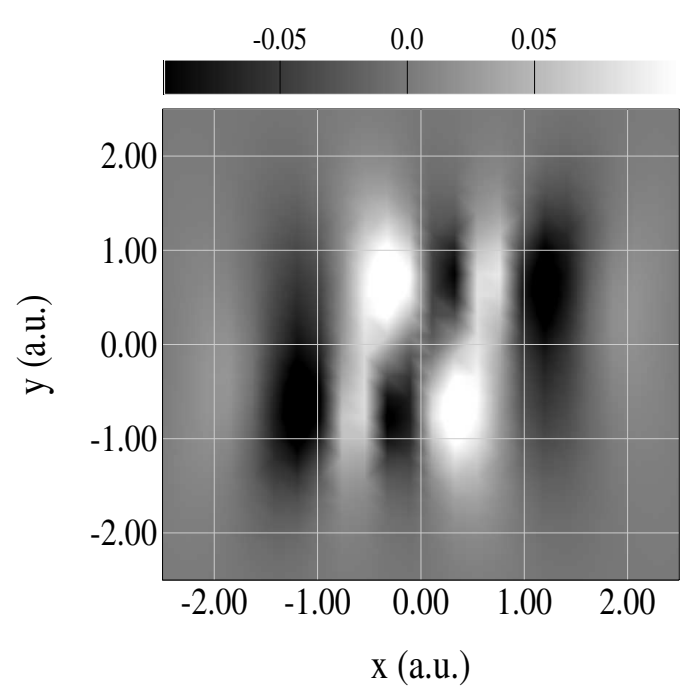

FIG. 4. Map of emission for the 79th harmonic generated by the $2 \mathrm{D} \mathrm{H}_{2}{ }^{+}$molecular ion in a $780 \mathrm{~nm}$ pulse of $5 \times$ $10^{14} \mathrm{~W} / \mathrm{cm}^{2}$ intensity. The alignment angle is $56^{\circ}$, very close to the angle minimizing the 79th harmonic amplitude, see Fig. 3(a).

position of the minimum does not depend on the laser parameters.

We proceed to analyze the harmonic emission into contributions from the various positions within the molecular ion. For radiation polarized along the $x$ axis, Eq. (4) can be rewritten as $S(\omega) \sim\left|\int a(\mathbf{r}, \omega) d^{2} r\right|^{2}$, where

$$
a(\mathbf{r}, \omega)=\int \varphi^{*}(\mathbf{r}, t)\left(\frac{\partial V(\mathbf{r})}{\partial x}+E(t)\right) \varphi(\mathbf{r}, t) e^{i \omega t} d t .
$$

Figure 4 is a density plot of the real part of the complex value $a(\mathbf{r}, \omega)$ for $2 \mathrm{D} \mathrm{H}_{2}{ }^{+}$. Here, we have employed the frequency $\omega$ of the 79th harmonic and an angle of $56^{\circ}$ which is very close to the critical angle for the 79th harmonic. It is obvious from the figure that different regions within the molecule may give positive or negative contributions. The sum of these contributions gives a very small total amplitude because, at the chosen angle, the extents of positive and negative areas are almost equal. If the angle of alignment is far from the critical angle, our calculation predicts that either the positive or the negative areas dominate, giving rise to a large total amplitude. The suppression of harmonic emission at a certain angle is thus an interference phenomenon. Plotting the imaginary part of $a(\mathbf{r}, \omega)$ leads to the very same conclusions. Furthermore, the same analysis can be applied to the numerical collision experiment described above, giving very similar results and confirming that the recollision picture is applicable. The phase jumps are easily interpreted within the interference picture: At angles below the critical angle, the total harmonic phase is given by the harmonic phase in the dominant regions of the molecule. When the angle gets closer to the critical angle, the regions giving contributions of opposite phase grow, and beyond the critical angle, they become dominant and thus determine the total phase.

Destructive interference has also been proposed [16] as the reason for the suppression of multiphoton ionization in the $\mathrm{O}_{2}$ molecule [17]. However, the origin of the phase mismatch in $\mathrm{O}_{2}$ lies completely in the antibonding symmetry of the valence orbital, while in the present case it arises from the wave function of the recolliding electron.

In summary, we have shown that high-harmonic generation by small molecules is very sensitive to the angle between the molecular axis and the polarization axis. The harmonic spectra exhibit minima which are characteristic of the molecule and independent of the laser parameters. They originate from interference between contributions from different locations within the molecule. This effect is very general and is expected to appear in many other molecules.

This work was supported by the U.K. Engineering and Physical Sciences Research Council, the EU "COCOMO" network (EU-IHP HPRN-CT-1999-0129), and by EU-IHP HPMF-CT-1999-00346.

*Present address: Instituto Nazionale Fisica della MateriaDipartimento Scienze Fisiche, Via Cintia, 26-80126Napoli, Italy.

[1] A. McPherson et al., J. Opt. Soc. Am. B 4, 595 (1987).

[2] A. L'Huillier, K. J. Schafer, and K. C. Kulander, J. Phys. B 24, 3315 (1991).

[3] M. Protopapas, C. H. Keitel, and P. L. Knight, Rep. Prog. Phys. 60, 389 (1997).

[4] P. Salières, A. L'Huillier, P. Antoine, and M. Lewenstein, Adv. At. Mol. Opt. Phys. 41, 83 (1999).

[5] P. B. Corkum, Phys. Rev. Lett. 71, 1994 (1993).

[6] G. G. Paulus et al., Phys. Rev. Lett. 72, 2851 (1994).

[7] M. Yu. Ivanov and P. B. Corkum, Phys. Rev. A 48, 580 (1993); T. Zuo, S. Chelkowski, and A. D. Bandrauk, ibid. 48, 3837 (1993).

[8] R. Kopold, W. Becker, and M. Kleber, Phys. Rev. A 58, 4022 (1998).

[9] A. D. Bandrauk and H. Yu, Phys. Rev. A 59, 539 (1999).

[10] T. Kreibich, M. Lein, V. Engel, and E. K. U. Gross, Phys. Rev. Lett. 87, 103901 (2001).

[11] D. G. Lappas and J. P. Marangos, J. Phys. B 33, 4679 (2000).

[12] R. Velotta et al., Phys. Rev. Lett. 87, 183901 (2001).

[13] "Vertical" ionization potential refers to fixed internuclear distance.

[14] M. D. Feit, J. A. Fleck, Jr., and A. Steiger, J. Comput. Phys. 47, 412 (1982).

[15] B. Sundaram and P. W. Milonni, Phys. Rev. A 41, 6571 (1990); K. Burnett, V. C. Reed, J. Cooper, and P. L. Knight, ibid. 45, 3347 (1992); J. H. Eberly and M. V. Fedorov, ibid. 45, 4706 (1992); D. G. Lappas, M. V. Fedorov, and J. H. Eberly, ibid. 47, 1327 (1993).

[16] J. Muth-Böhm, A. Becker, and F. H. M. Faisal, Phys. Rev. Lett. 85, 2280 (2000).

[17] A. Talebpour, C.-Y. Chien, and S. L. Chin, J. Phys. B 29, L677 (1996); C. Guo, Phys. Rev. Lett. 85, 2276 (2000). 